\title{
Desarrollo de un Recubrimiento Comestible Compuesto para la Conservación del Tomate de Árbol (Cyphomandra betacea S.)
}

\author{
Johana C. Andrade, Diana L. Acosta, Mauricio A. Bucheli y Oswaldo Osorio \\ Universidad de Nariño, Facultad de Ingeniería Agroindustrial, Grupo de investigación Tecnologías \\ Emergentes en Agroindustria (TEA) Grupo De Apoyo a la investigación y Desarrollo Agroalimentario \\ GAIDA, Ciudadela Universitaria Torobajo, San Juan de Pasto-Colombia. \\ (e-mail: joha.intel0705@hotmail.com; alvear_diana@hotmail.com; mauricio_bucheli@yahoo.com; \\ osorio_oswaldo@hotmail.com)
}

Recibido Ene. 16, 2014; Aceptado Mar. 11, 2014; Versión final recibida May. 10, 2014

\section{Resumen}

Se estudió la viabilidad de utilizar cera de laurel (Morella pubescens H\&B ex Willd-Wilbur), como base de un recubrimiento comestible capaz de prolongar la vida útil del tomate de árbol (Cyphomandra betacea S.). Para ello se evaluaron nueve formulaciones, teniendo en cuenta la pérdida de peso de las diferentes muestras mediante un diseño factorial $3^{2}$, aleatorizado por bloques, analizado con el programa estadístico Statgraphics Centurion XVI.II. Esto dio lugar a la optimización de la formulación, obteniendo un recubrimiento compuesto que se evaluó sobre las propiedades fisicoquímicas y sensoriales de los frutos. El análisis estadístico mostró que el recubrimiento redujo la pérdida de masa y el índice de respiración manteniendo la firmeza y la calidad sensorial del fruto por más tiempo, incrementando en un $25 \%$ el tiempo de anaquel del tomate de árbol con respecto a las muestras testigos sin recubrimiento.

\section{Development of an Edible Coating Compound for the Conservation of Tree Tomato (Cyphomandra betacea S.)}

\begin{abstract}
The viability of using laurel wax (Morella pubescens H\&B ex Willd-Wilbur) as an edible coating base which is able to extend the useful life of tree tomatoes (Cyphomandra betacea S.) was studied. To achieve this, new formulations were analyzed considering the mass loss of different samples through a factorial design $3^{2}$, randomized in blocks. These samples were analyzed with the help of the statistical software Statgraphics Centurion XV. This analysis allowed the optimization of the formulation, thus obtaining a coating compound that was evaluated in relation to the physicochemical and sensorial properties of the fruits. The analysis showed that the covering reduced the mass loss and the breathing indicator, and maintained the sensorial quality and firmness of the fruits for longer time. The storing time of the tree tomatoes increased by $25 \%$ in relation to the samples, without coating.
\end{abstract}

Keywords: edible covering, laurel wax, tomato tree, conservation 


\section{INTRODUCCIÓN}

El creciente deterioro ambiental asociado con el uso masivo de materiales plásticos derivados del petróleo en el empaque y embalaje de los alimentos, ha propiciado la búsqueda de nuevas alternativas para su sustitución, cuyas fuentes sean materiales renovables, biodegradables y de origen natural (Velazco et al., 2012), este interés se centra además en compuestos que interaccionen efectivamente con el producto, que permitan una modificación adecuada de los gases internos, y sobre todo, que no modifiquen las características sensoriales del mismo (Guilbert et al., 1996). Es así como en los últimos años se ha incrementado el uso de diferentes recubrimientos comestibles, como alternativa de envasado natural, convirtiéndose en una técnica innovadora para la conservación de la calidad de los alimentos, en especial de productos altamente perecederos, como los pertenecientes a la cadena hortofrutícola, los cuales, presentan un deterioro progresivo tras la cosecha debido a fenómenos fisiológicos como transpiración y respiración, que conllevan daños en la calidad de los mismos. De este modo, el mecanismo de acción de los recubrimientos busca constituir una barrera semipermeable al vapor de agua, al oxígeno y al dióxido de carbono, retrasando el deterioro y manteniendo la integridad estructural del producto (Navarro, 2007).

La implementación de esta tecnología es un campo prometedor, aun sin explorar a plenitud, que implica la búsqueda de productos con potencial para llegar a ser parte de la matriz estructural de este tipo de recubrimientos, dentro de este grupo se encuentran polisacáridos, proteínas, lípidos, entre otros. Estos pueden actuar como constituyente único o manejarse en mezclas, todo con el fin de aprovechar las propiedades de cada compuesto y la sinergia entre ellos, para lograr las mejores propiedades mecánicas y de barrera, ya que estas dependen directamente de los compuestos que integran la matriz (Altenhofen et al., 2009). Cada tipo de recubrimiento tiene características propias definidas por su composición, es así como los carbohidratos son excelentes fuentes de biopolímeros, además su bajo precio y accesibilidad despierta un gran interés a nivel mundial, reflejado en diversas investigaciones que han comprobado su efectividad, entre ellos, el almidón de yuca ha tenido gran acogida debido a que presenta buen aspecto, no es pegajoso, es un recurso de alta disponibilidad en diversas partes del mundo, es brillante y transparente, mejora el aspecto visual de la fruta y puede ser removido con agua, lo que representa una alternativa potencial para ser utilizado en la conservación de frutas y hortalizas (Bengtsson et al., 2003; Medina y Salas, 2008; Bemiller y Whistler, 2009; Ruiz et al., 2009), además es una de las materias primas más utilizadas en la formación de empaques naturales debido a su capacidad de gelificar, moldear y formar películas (Enríquez et al., 2012a).

Los polisacáridos y las proteínas son buenos materiales para la formación de recubrimientos, ya que muestran excelentes propiedades mecánicas y estructurales (Chambi y Grosso, 2006; Ponce et al., 2008; Altenhofen et al., 2009; Bosquez et al., 2010; Enríquez et al., 2012b), pero presentan una pobre capacidad de barrera frente a la humedad, este problema no se encuentra en los lípidos dadas sus propiedades hidrofóbicas, especialmente en los que poseen puntos de fusión altos tales como la cera de abejas y la cera carnauba (Morillon et al., 2002; Caceres et al., 2003), sin embargo ellos presentan malas propiedades mecánicas, que deben contrarrestarse con el uso de aditivos (García et al., 2000; Mchug et al., 2000; Panuwat et al.,2008). Las propiedades mecánicas definen la capacidad de los recubrimientos de formar capas uniformes y estables que se adapten a los cambios de volumen generados por la deshidratación que experimentan los productos que protegen, es por ello que para contrarrestar las deficiencias mecánicas de los lípidos, se han realizado formulaciones en las que se incluye un material hidrocoloide y aditivos como plastificantes, emulsificantes y tensoactivos, dando lugar a recubrimientos compuestos (Bureau y Multon, 1995; Chiumarelli, 2014).

Un material lipídico interesante para la formación de recubrimientos es la cera de laurel, producto que se obtiene del fruto maduro del Laurel (Morella pubescens H\&B ex Willd-Wilbur), arbusto de origen holártico, que se encuentra distribuido en Colombia, Ecuador, Perú, Venezuela, Bolivia y Costa Rica (Arango et al., 2009). La extracción de esta cera es un proceso que aún se realiza de manera tradicional en la mayoría de lugares donde se produce; en el departamento de Nariño, Colombia la obtención se efectúa por prensado, disponiendo el fruto precalentado (contenido en sacos de fibra de fique elaborados en un tejido especial para este fin) en prensas de tornillo durante veinte minutos, tiempo tras el cual se obtiene cera semilíquida que se vierte en moldes para su posterior almacenamiento y distribución (Muñoz y Luna, 2002).

Medina et al. (2003) determinaron la composición de la cera de laurel (Myrica pubescens) empleando las técnicas de cromatografía de columna (Fase estacionaria: sílica gel), extracción en fase sólida (SPE) y derivatización con $\mathrm{BF}_{3}$, esta última para obtener los ésteres de los respectivos ácidos grasos presentes en la cera. Para la elución de la muestra se empleó dicloromentano, hexano y una mezcla de los dos en relaciones adecuadas. Cada una de las fracciones obtenidas se analizaron por cromatografía de gases acoplada a espectrometría de masas (GC/SM) encontrando como componentes mayoritarios el ácido mirístico en una proporción del 45,65 \% y el ácido palmítico en una proporción de 45,28 \% y como 
componentes minoritarios los ácidos laúrico, 12-metiltridecanoico, 2-metiltetradecanoico, 3metilpentadecanoico, esteárico, 13-metiltetradecanoico y eicosanoico. De igual manera se determinó que el glicerol hace parte de la composición química de la cera a través de espectroscopia Infrarroja (IR).

La composición global de la cera de laurel refleja su inocuidad, siendo un producto con potencial para ser utilizada sobre alimentos. Sin embargo, su carácter altamente apolar hace que la solubilidad disminuya a medida que aumenta la polaridad de los solventes, siendo di-clorometano, éter etílico y hexano algunos de componentes en que la cera se solubiliza (Medina et al.,2003). Por tanto, para poder realizar mezclas en agua es necesario utilizar un emulsificante que permita la disolución. Estas características proporcionan una buena barrera frente a la pérdida de agua, pero generan deficiencias en las propiedades mecánicas, como baja elasticidad, resistencia y flexibilidad, dificultando la viabilidad de los recubrimientos (Mosquera et al., 2002). Bajo este contexto, la presente investigación buscó la obtención de un recubrimiento a base de cera de laurel, capaz de conservar la calidad del tomate de árbol (Cyphomandra betacea S.), mediante la formulación de un recubrimiento compuesto que contrarreste las deficiencias mecánicas de la cera de laurel y cuya funcionalidad sea comprobada fisicoquímica y sensorialmente a lo largo del tiempo de vida útil del fruto.

\section{MATERIALES Y MÉTODOS}

Los materiales empleados en la elaboración del recubrimiento comestible fueron: cera de laurel donada por el grupo de investigación PIFIL de la Universidad de Nariño, agua, almidón de yuca comercial, propilenglicol y glicerol, como plastificantes, Tween 80 (monoestearato de sorbitan etoxilado) como emulsificante y como tensoactivos aceite de oliva y glucosa. Se empleó tomate de árbol en estado de madurez tres, según la Norma Técnica Colombiana NTC 4105, obtenido de un mismo proveedor en un mercado local de la ciudad de Pasto-Nariño, Colombia. Los frutos se sometieron a operaciones de limpieza, desinfección, selección y clasificación.

\section{Determinación de la formulación del recubrimiento comestible}

Se estudiaron nueve formulaciones, compuestas por una mezcla base, que se mantuvo constante ( $3 \mathrm{~g}$ de cera de laurel, $0.5 \mathrm{~g}$ de aceite de oliva, $0.2 \mathrm{~g}$ de Tween $80,0.7 \mathrm{~g}$ de propilenglicol, $1 \mathrm{~g}$ de glicerol y $0.2 \mathrm{~g}$ de glucosa), y una matriz hidrocoloide formada por almidón y agua, cuyas cantidades fluctuaron dentro de límites funcionales establecidos mediante pruebas preliminares tomando como punto de partida la mezcla base. Los límites para el almidón corresponden a cantidades que se encuentran entre $0.5 \mathrm{y} 2.5 \mathrm{~g}$, y para el agua entre 5 y $45 \mathrm{~g}$, dentro de los cuales se establecieron tres niveles a evaluar, codificados como $-1,0$ y 1 , representando una cantidad menor, intermedia y mayor, respectivamente.

Todos los recubrimientos se prepararon siguiendo la misma metodología; el primer paso fue la dilución del almidón de yuca en agua, mezcla que se llevó a $70{ }^{\circ} \mathrm{C}$ bajo agitación constante (1200 rpm) hasta alcanzar la gelificación o coagulación térmica, mecanismo de elaboración de la matriz hidrocoloide del recubrimiento, luego se adicionó la cera de laurel y el aceite de oliva, seguido por Tween 80, propilenglicol, glicerol, y glucosa, en su orden, la agitación continuó durante 15 minutos más, tiempo en el cual se consiguió una mezcla homogénea y estable, resultado de la interacción generada entre los componentes no polares que conforman los diferentes tratamientos, la acción emulsificante del Tween 80 que actúa reduciendo la tensión superficial entre compuestos insolubles y la fuerza mecánica de la agitación que juega un papel vital en la emulsificación de las soluciones formadoras de recubrimientos.

Los diferentes revestimientos elaborados se dispersaron uniformemente sobre la superficie de la fruta, logrando una capa suficientemente fina que no es detectada a simple vista por el consumidor. La optimización de la formulación y el análisis estadístico, se realizó mediante un diseño factorial $3^{2}$, aleatorizado por bloques con 4 repeticiones por cada una de las nueve opciones, teniendo un total de 36 muestras experimentales, analizadas con el programa Statgraphics Centurion XVI.II. La variable respuesta estudiada, fue la pérdida de peso de los frutos, obtenida por el método de gravimetría, factor vital y determinante en la calidad organoléptica y fisicoquímica del tomate de árbol. El recubrimiento con la formulación más efectiva, se caracterizó a nivel fisicoquímico, a través de análisis realizados por triplicado, para los parámetros: densidad, viscosidad (viscosímetro digital Visco Basic Plus, FUNGILAB, T: $20 \stackrel{\circ}{\circ}$ Aguja N. R3 a 60 rpm), pH (pH-metro HANNA HI 8314), humedad y cenizas (Método de desecación $100{ }^{\circ} \mathrm{C}$ por 3 horas y $700{ }^{\circ} \mathrm{C}$ por 15 minutos, respectivamente).

\section{Evaluaciones realizadas sobre el tomate de árbol}

Tras la optimización de la formulación, se comprueba su efectividad sobre la calidad del tomate de árbol (Cyphomandra betacea S.) mediante pruebas por triplicado realizadas cada cuatro días en condiciones ambientales ( $\mathrm{T}: 18+/-2^{\circ} \mathrm{C}$ y HR:68 \%), para pérdida de peso, dureza, $\mathrm{pH}$, acidez titulable, sólidos solubles 
totales e intensidad respiratoria; mientras que la transpiración, se evaluó por cinco días seguidos en tres ambientes diferentes.

Pérdida de peso: se evaluó por el método gravimétrico, utilizando una balanza analítica ADAM con precisión de $0,1 \mathrm{mg}$.

Dureza: la dureza de los frutos se determinó utilizando un penetrómetro BISHOP FT 327, con una punta de $5 \mathrm{~mm}$ de diámetro.

pH del zumo: se evaluó con un pH metro HANNA HI 8314, previamente calibrado con tampones a pH 4 y 7.

Sólidos solubles: determinado mediante el uso del refractómetro ATAGO ATC-1 (0-32 ํx a $\left.18{ }^{\circ} \mathrm{C}\right)$.

Acidez titulable: se obtuvo por titulación con $\mathrm{NaOH} 0,1 \mathrm{~N}$ y fenoltaleína como indicador, mediante una bureta digital marca JENCONS DIGITRATE PRO $50 \mathrm{ml}$.

Intensidad respiratoria: se determinó en un ambiente previamente purgado y herméticamente cerrado, utilizando el Medidor de $\mathrm{CO}_{2}$ Lutron GC 2028.

Transpiración: se realizó en tres niveles: refrigeración (T:4 $\left.{ }^{\circ} \mathrm{C}, \mathrm{HR}: 68 \%\right)$, incubación $\left(\mathrm{T}: 26{ }^{\circ} \mathrm{C}, \mathrm{HR}: 55 \%\right)$, y ambiente $\left(\mathrm{T}: 18{ }^{\circ} \mathrm{C}, \mathrm{HR}: 68 \%\right)$, y fue evaluada por gravimetría, incluyendo el índice de transpiración en su determinación, con el fin de probar el comportamiento del recubrimiento sobre los frutos en diferentes condiciones ambientales.

Diseño experimental: se realizó un diseño experimental de un factor categórico por comparación de muestras, mediante la diferencia de medias de Fisher con $95 \%$ de confiabilidad, analizado con el programa estadístico Statgraphics Centurion XVI.II.

Evaluación sensorial de los frutos: se realizó la evaluación sensorial de muestras de tomates recubiertos y testigos. A los diez días de seguimiento con el apoyo de quince jueces consumidores del fruto, a través de tres pruebas: prueba de preferencia para las características brillo, color, textura y la apariencia general; prueba discriminativa triangular, para determinar la existencia de diferencias significativas entre las muestras y prueba de clasificación por medio de escalas de intervalo, para evaluar el olor, a través de una escala de cuatro puntos. La interpretación de resultados se dio porcentualmente y por medio de tablas de interpretación estadística de Roessler et al., (1948) citado por Anzaldua (1994).

\section{RESULTADOS Y DISCUSIÓN}

\section{Formulación del recubrimiento}

La Figura 1 demuestra que el ALMIDÓN $(A)$, como componente individual dentro de la formulación no presenta un efecto significativo sobre la variable respuesta, pérdida de peso, a diferencia del AGUA (B) y la interacción ALMIDÓN-AGUA (AB), que tienen un efecto inversamente proporcional sobre este parámetro, además la figura refleja la importancia de la relación $A B$ en la reducción de la pérdida de peso, demostrando que la matriz hidrocoloide, formada por estos dos componentes es vital para la funcionalidad del recubrimiento.

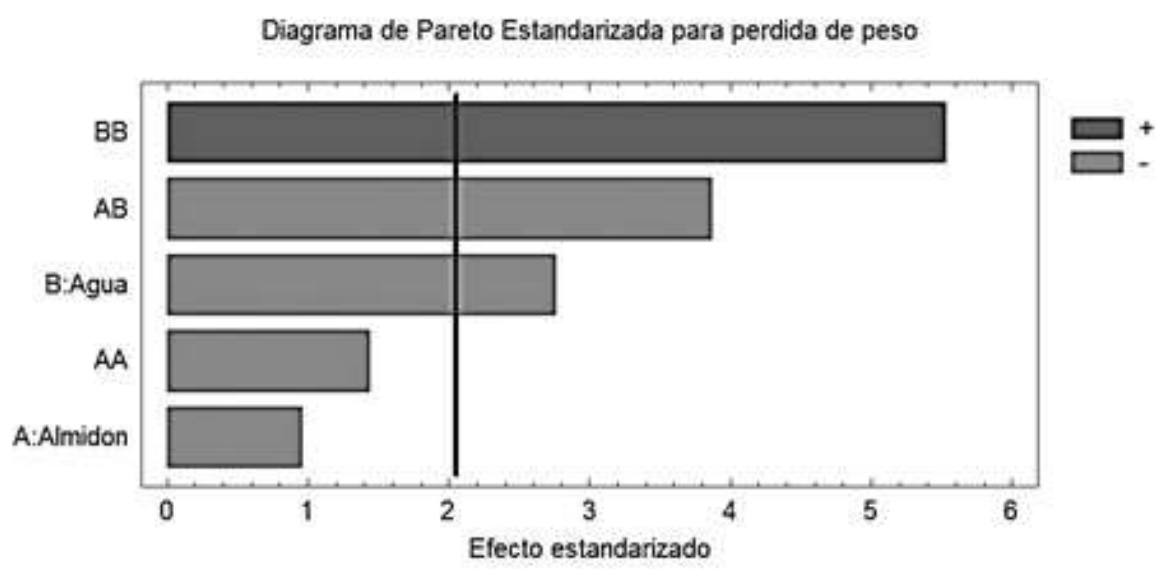

Fig. 1: Diagrama de Pareto estandarizado para porcentaje pérdida de peso (nivel de confianza 95\%) 
La superficie de respuesta estimada, Figura 2., muestra la interacción de los dos factores estudiados y el efecto conjunto que ejercen sobre el porcentaje de pérdida de peso de los frutos de tomate de árbol, de modo que cuando las formulaciones del recubrimiento toman cantidades de agua correspondientes al límite inferior o superior dentro del intervalo funcional, la pérdida de peso se eleva, sin importar la cantidad de almidón con la cual se trabaje (dentro de los niveles estudiados). La superficie de respuesta, además, conlleva un proceso de optimización, que indica que para minimizar la pérdida de peso en los frutos, los factores almidón y agua deben trabajarse en niveles 1 y 0.39 respectivamente, equivalentes a $4.5 \mathrm{~g}$ de almidón y $32.8 \mathrm{~g}$ agua.

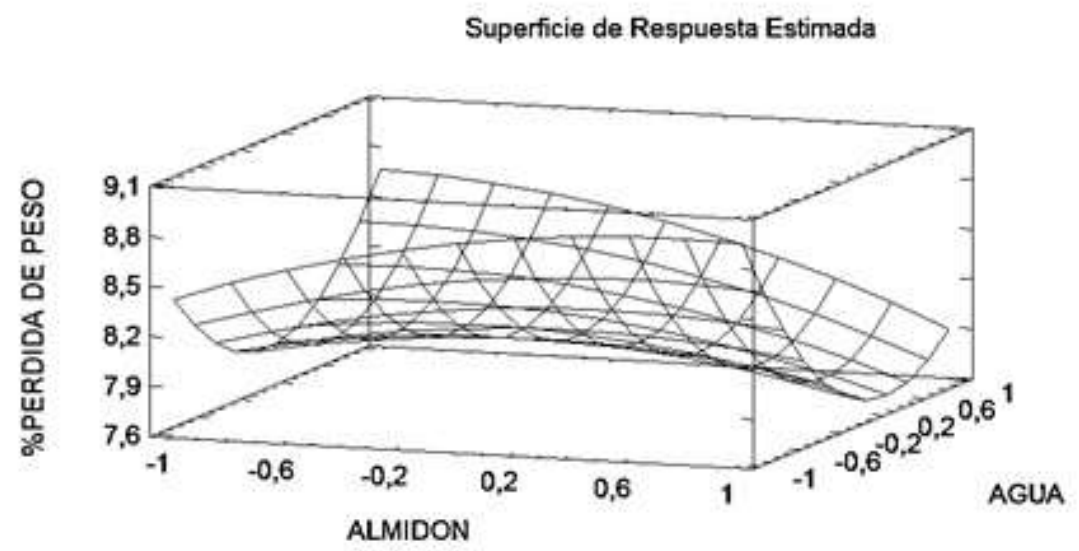

Fig. 2: Superficie de respuesta estimada para porcentaje pérdida de peso

\section{Caracterización del recubrimiento alimentario}

El recubrimiento obtenido presentó valores de densidad de $0.95 \mathrm{~g} / \mathrm{ml}\left(20{ }^{\circ} \mathrm{C}\right)$ y un valor de $\mathrm{pH}$ de 6.4 , similares a los reportados por Navarro et al. (2007), en recubrimientos elaborados con hidroxipropilmetilcelulosa y cera de abejas. La viscosidad obtenida (952.9 cP) y la densidad, presentaron valores elevados que se deben al alto contenido de hidrocoloide, reafirmando así la teoría de Parzanese et al. (2010), quien manifestó que al utilizar una cantidad considerable de hidrocoloide en la formulación aumenta la proporción de estos parámetros. El porcentaje de humedad (54.3\%), se debe a la base acuosa del hidrocoloide, mientras que el porcentaje de cenizas $(0.56 \%)$, indica los minerales presentes en el recubrimiento.

\section{EVALUACIÓN DEL RECUBRIMIENTO SOBRE EL TOMATE DE ÁRBOL}

\section{Pérdida de peso y dureza}

Los resultados obtenidos revelaron que el revestimiento redujo en un $30 \%$ la pérdida de peso, como se observa en la Figura 3; además conservó por cinco días más la dureza de los tomates recubiertos con respecto a los testigos. Estos resultados coinciden con los obtenidos por Heredia et al. (1999) y Báez et al. (2002), quienes encontraron que el uso de cubiertas cerosas reduce la pérdida de peso y aumenta la dureza. Con ello se demuestra que el recubrimiento crea una barrera efectiva frente a la pérdida de agua, evitando de esta manera el deterioro de la fruta y la reducción de la calidad, este fenómeno está ligado a la cera de laurel, material lipídico hidrofóbico vital para la efectividad del revestimiento.

Sólidos solubles ( ${ }^{\circ}$ Brix), pH y acidez titulable.

Los parámetros de calidad correspondientes a sólidos solubles (S.S.), pH y acidez titulable, presentaron una tendencia inherente a la maduración de los frutos, razón que justifica la disminución del contenido de ácido cítrico y por consiguiente el incremento del pH y sólidos solubles con el transcurso de los días.

Los tomates recubiertos mostraron una reducción en S.S. respecto a los testigos, tendencia relacionada con el retardo del proceso de maduración en las muestras con recubrimiento, comportamiento observado anteriormente por Castricini, (2009) y Chiumarelli (2014). Mientras que los valores de pH y acidez titulable no fueron afectados por el recubrimiento, resultados similares se presentaron en estudios previos realizados por Bósquez (2003) y Mulkay et al. (2004), quienes señalan que factores como acidez titulable y pH, no son afectados por la mayoría de los recubrimientos comestibles que se han investigado. 


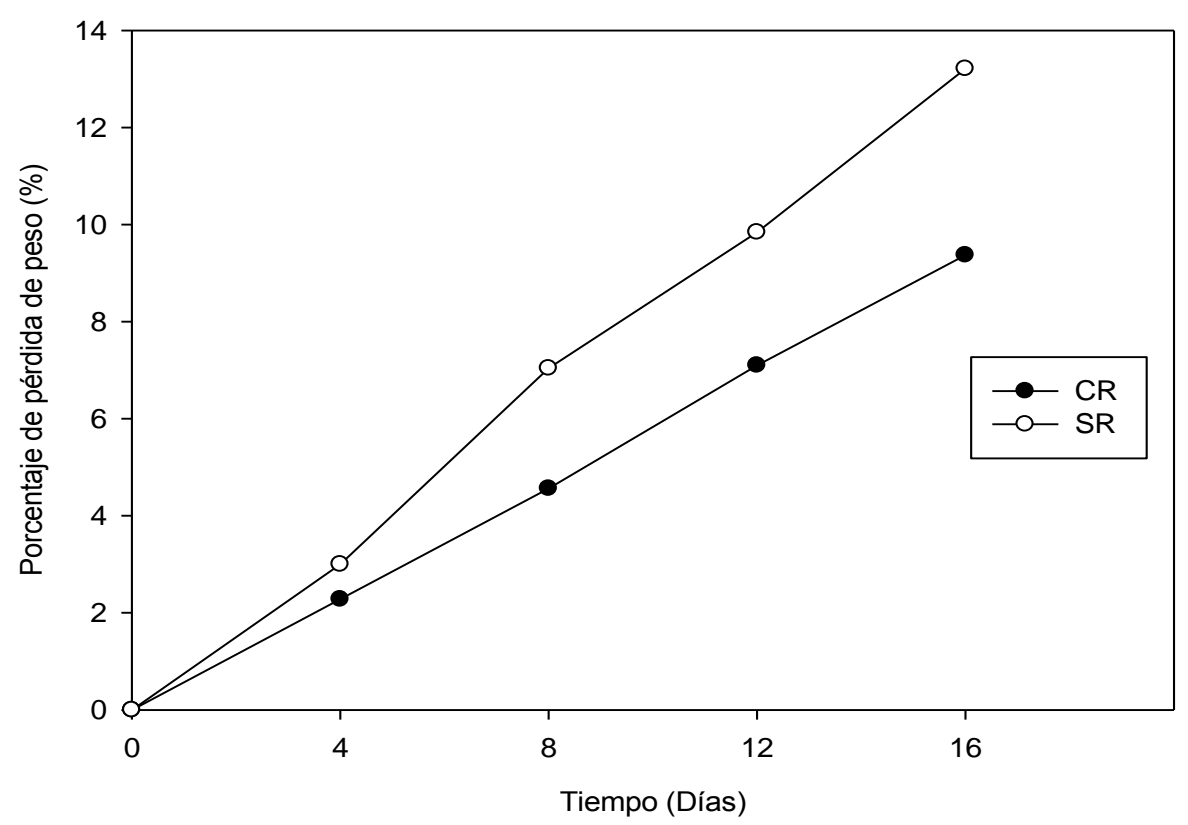

Fig. 3: Pérdida de peso para tomates de árbol (Cyphomandra betacea S.) con recubrimiento (CR) y sin recubrimiento (SR).

Índice de respiración

El comportamiento del índice de respiración en los tomates con y sin recubrimiento, ratifica su condición como fruto no climatérico, como se observa en la Figura 4., donde la respiración desciende en forma progresiva hasta el día doce, cuando los tomates testigos empiezan a presentar una estabilidad correspondiente a los días de sobre maduración, esto trae consigo que los atributos de consumo empiecen afectarse; mientras que en los tomates recubiertos aún no se percibe la estabilidad, lo cual se refleja en una apariencia agradable respecto al testigo.

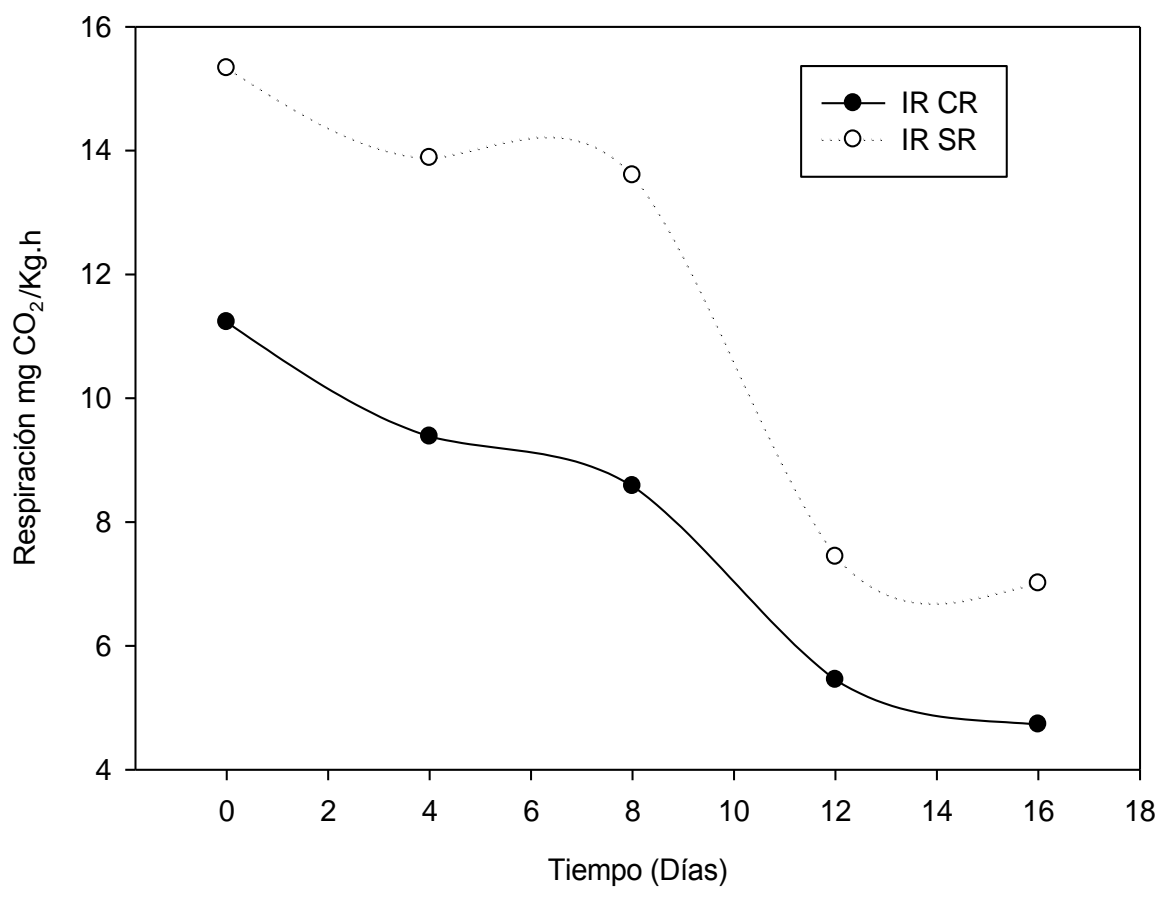

Fig. 4: Índice de respiración (IR) para tomates de árbol (Cyphomandra betacea S.) con recubrimiento (CR) y sin recubrimiento $(\mathrm{SR})$. 
Estos resultados indican la capacidad del recubrimiento de generar un efecto similar al de las atmósferas modificadas, gracias a la barrera semipermeable al dióxido de carbono y al oxígeno, generada por sus componentes, haciendo que los tomates con el revestimiento disminuyan la intensidad respiratoria, conservando su calidad fisicoquímica y sensorial por un tiempo mayor que los testigos, estos resultados se pueden comparar con los obtenidos por Navarro (2007), Restrepo (2009) y Chiumarelli et al. (2011) quienes reportaron comportamientos similares para ciruela, fresa y mango Tommy respectivamente.

\section{Transpiración}

La figura 5, revela el comportamiento de la transpiración de los tomates recubiertos y los testigos, expuestos a diferentes condiciones ambientales; en todos los casos se observa que los tomates con el recubrimiento presentaron un valor menor para este parámetro frente a los testigos. La reducción obtenida fue de un 26,5 $\%$ en refrigeración, de un $25 \%$ en condiciones ambientales y de $40 \%$ en incubación, datos que reflejan una diferencia significativa entre los tomates recubiertos y testigos.

Los resultados muestran que el recubrimiento conserva su funcionalidad dentro de un rango amplio de temperaturas, por lo tanto puede ser utilizado en tomates de árbol que se sometan a refrigeración o aquellos que deban soportar temperaturas altas.

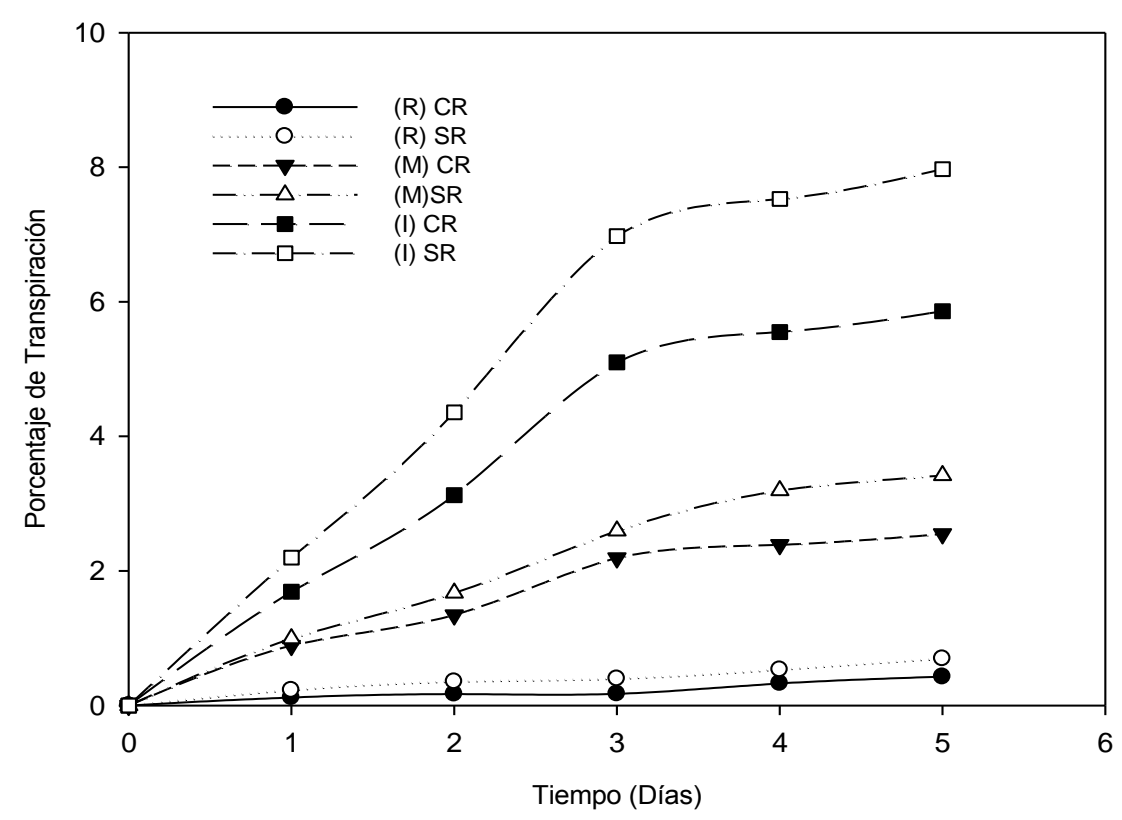

Fig. 5: Transpiración del tomate de árbol con recubrimiento $(\mathrm{CR})$ y sin recubrimiento $(\mathrm{SR})$ en condiciones ambientales (M), refrigeración (R) e incubación (I).

\section{EVALUACIÓN SENSORIAL DE LOS FRUTOS}

\section{Prueba de preferencia}

Los resultados de esta prueba indicaron que para las características de brillo y textura el $100 \%$ de los jurados prefirieron los tomates recubiertos, con un nivel de significancia del $0,1 \%$, mientras que para color, la preferencia fue del $87 \%$, con un nivel de significancia del $1 \%$.

\section{Prueba discriminativa triangular}

Los resultados mostraron que el $87 \%$ de los jurados determinaron correctamente la muestra diferente, con un nivel de significancia del $0,1 \%$, que indica la existencia de una diferencia evidente a nivel sensorial que hace posible distinguir entre las dos muestras, debido a la buena apariencia de los tomates recubiertos factor ligado al carácter protector del revestimiento.

\section{Prueba de clasificación por escalas de intervalo}

El $60 \%$ de los jueces no percibieron ningún olor en las muestras de tomates recubiertos, mientras que el 40 $\%$ restante detecto un leve olor inherente a la fruta, lo cual demuestra que el recubrimiento retiene los compuestos volátiles sin generar ningún tipo de alteración en el olor característico del tomate. 
Todas las pruebas sensoriales mostraron que el recubrimiento conserva la calidad visual del tomate de árbol por mayor tiempo, esto sin modificar factores como el olor o el color, resultados similares a los recubrimientos elaborados a partir de cera de abejas o carnauba reportados por Morillon et al. 2002 y Caceres et al. 2003 respectivamente.

\section{CONCLUSIONES}

El recubrimiento obtenido se denomina "compuesto" ya que su matriz estructural se compone de cera de laurel, material lipídico capaz de brindarle al recubrimiento una barrera efectiva contra la pérdida de agua; almidón como hidrocoloide, que permite constituir una barrera semipermeable al oxígeno y al dióxido de carbono y aditivos, vitales en el mejoramiento de las propiedades mecánicas, generando un revestimiento funcional y estable.

El recubrimiento resultó una buena alternativa de conservación del tomate de árbol (Cyphomandra betacea S.) debido a sus excelentes propiedades de barrera frente a la transferencia de agua, las cuales se ven reflejadas en la reducción de la pérdida de peso, una mayor firmeza y una buena apariencia, lo que llevó a los frutos recubiertos a incrementar su vida de anaquel en un $25 \%$ más con respecto a las muestras testigo expuestas a condiciones similares $\left(\mathrm{T}: 18^{\circ} \mathrm{C}+/-2^{\circ} \mathrm{C}, \mathrm{HR}: 68 \%\right)$.

El índice de respiración presentado por el tomate de árbol (Cyphomandra betacea S.) se redujo debido a la acción semipermeable del recubrimiento frente a los gases como $\mathrm{O}_{2}$ y $\mathrm{CO}_{2}$, factor vital ya que al trabajar con películas totalmente impermeables se impide la entrada y salida de gases generando reacciones anaerobias que se traducen en alteraciones de las frutas como aparición de olores desagradables o maduración anormal.

Se determinó que el recubrimiento no genera alteraciones en las propiedades organolépticas de los frutos recubiertos, además el revestimiento brindó a los frutos características más agradables para el consumidor referentes a brillo, color, olor, textura y apariencia con respecto a los tomates sin recubrimiento.

\section{REFERENCIAS}

Altenhofen, M., Krause, A., Guenter, T., Alginate and pectin composite films crosslinked with Ca+2ions: Effect of the plasticizer concentration, Carbohydrate polymers, 77, 736-742 (2009)

Anzaldua, A., La evaluación sensorial de los alimentos en la teoría y la práctica., ACRIBIA, S.A., Zaragoza, España (1994)

Arango, O., Hurtado, A., Castillo, P., Santacruz, M., Estudio de las condiciones de extracción por arrastre con vapor del aceite esencial de laurel de cera (Morella pubescens), Revista Biotecnología en el Sector Agropecuario y Agroindustrial, ISSN: 1692-3561, 7(2), (2009)

Báez, R., Saucedo, C., Pérez, B., Bringas, E., Mendoza, A., Efecto de la aplicación de cera comestible y agua caliente sobre la conservación de melón reticulado, Fitotecnia Mexicana, 25(4), 375-379 (2002)

Bemiller, J. and Whistler, R., Starch chemistry and technology, Amsterdam (Netherlands): Academic Press, 3 (2009)

Bengtsson, M., Koch, K., Gatenholm, P., Surface octanoylation of high amylose potato starch films, Carbohydrate Polymers, 54, 1-11 (2003)

Bósquez, E., Elaboración de recubrimientos comestibles formulados con goma de mezquite y cera de candelilla para reducir la cinética de deterioro en fresco del limón persa (Citrus latifolia tanaka), Tesis doctoral en Ciencias Biológicas, Dpto. Ciencias Biológicas y de la Salud, Universidad Autónoma Metropolitana, México, D.F. (2003)

Bosquez M., Tomás, S., Rodríguez, M., Influence of $\mathrm{CaCl}_{2}$ on the water vapor permeability and the surface morphology of mesquite gumbased edible films, LWT-Food Science and Technology, 43, 1419- 1425 (2010)

Bureau, G. Multon, J., Embalaje de los alimentos de gran consumo., ACRIBIA SA. Zaragoza, España (1995)

Caceres, I., Martinez, J., Cuquerella, J. Del Rio, M., Navarro, P., Influencia del encerado en la calidad de la mandarina "Clemenules" procedente de sistemas de producción integrada, Rev. Iberamericana de tecnología postcosecha, 5, 113-116 (2003)

Castricini, A., Aplicação de revestimentos comestíveis para conservação de mamões (Carica papaya L.) 'Golden', Tese Doutorado em Fitotecnia, Departamento de Fitotecnia, Instituto de Agronomía, Universidade Federal Rural do Rio de Janeiro, Rio de Janeiro-Brasil (2009) 
Chambi, H., Grosso, C., Edible films produced with gelatin and casein cross-linked with trans glutaminase, Food Research International, 39, 458-456 (2006)

Chiumarelli, M., Ferrari, C., Sarantopoulos, C., Hubinger, M., Fresh cut 'Tommy Atkins' mango pre-treated with citric acid and coated with cassava (Manihot esculenta Crantz) starch or sodium alginate, Innovative Food Science \& Emerging Technologies, 12(3), 381-387 (2011)

Chiumarelli, M., Hubinger, M., Evaluation of edible films and coatings formulated with cassava starch, glycerol, carnauba wax and stearic acid, Food hydrocolloids,38 (2014)

Enríquez, M., Velasco, R., Fernández, A., Caracterización de almidones de yuca nativos y modificados para la elaboración de empaques biodegradables, Revista de biotecnología en el sector agropecuario y agroindustrial, 10(1), 162-174 (2012a)

Enríquez, M., Velasco, R., Ortiz, V., Composición y procesamiento de películas biodegradables basadas en almidón, Revista de biotecnología en el sector agropecuario y agroindustrial, 10(1), 182-192 (2012b)

García, M. Martino, M. And Zaritzky, N., Lipid addition to improve barrier properties of edible starch-based films and coatings, Journal of Food Science, 65, 941-947 (2000)

Guilbert, S., Gontard, N., \& Gorris, L., Prolongation of the shelf-life of perishable food products using biodegradable films and coatings, Lebensmittel Wissenschaft and Technology, 29, 10-17 (1996)

Heredia, J., Contreras, L., Siller, J., Efectos del uso de ceras comestibles sobre la maduración postcosecha en papaya cv. Maradol, VIII Congreso de Horticultura, 216, Manzanillo-Colima, México (1999)

Medina, A., y Lopez, D., Caracterización química de los principales componentes de la cera de Laurel (Myricapubescens H.B.K.) que se cultiva en el departamento de Nariño, Tesis de pregrado, Facultad de ciencias Naturales y Matemáticas, Departamento de Química, Universidad de Nariño, Pasto-Colombia (2003)

Medina, J. y Salas, J., Caracterización morfológica del grano de almidón nativo: apariencia, forma, tamaño y su distribución, Revista de Ingeniería (Universidad de Los Andes, Bogotá, Colombia), 27, 56-62 (2008)

Mosquera, S. y Ortega, A., Evaluación de la cera de laurel (Myrica Pubescens) en el recubrimiento de quesos maduros, Tesis pregrado, Facultad Ciencias Pecuarias, Programa Zootecnia, Universidad de Nariño, Pasto-Colombia (2002)

Morillon, V., Debeaufort, F., Bond, G., Capelle, M.,Volley, A., Factors affecting the moisture permeability of lipid-based edible films, Critical Reviews in Food Science and Nutrition, 42(1), 67-89 (2002)

Mulkay T., Cáceres, R., Rodríguez, J., Paumier, A., Manejo de la maduración en frutos de papaya (Carica papaya L.) cv Maradol, Revista CitriFrut, 21, 9-13 (2004)

Muñoz, J. y Luna, C., Laurel de cera (Myrica pubescens H.B.K.), Universidad de Nariño, PIFIL, Casa editorial diario del sur, Pasto-Colombia, 105-112 (2002)

Navarro, M., Efecto de la composición de recubrimientos comestibles a base de hidroxipropilmetilcelulosa y cera de abeja en la calidad de ciruelas, naranjas y mandarinas, Tesis Doctoral, Dpt. Tecnología de Alimentos, Universidad Politécnica De Valencia, Valencia-España (2007)

Panuwat, S., Kees S., Stephen, B., Joseph, M., Efficacy of polythylene-based antimicrobial films containing principal constituents of basil, Elsevier, 41,779-788 (2008)

Ponce, A. y Roura S., Del Valle C., Moreira M., Antimicrobial and antioxidant activities of edible coating senriched with natural plant extracts: in vitro and in vivo studies, Postharvest Biology and Technology,49 (2), 294-300 (2008)

Restrepo, J. y Aristizábal I., Conservación de fresa (Fragaria x ananassa Duch cv. Camarosa) mediante la aplicación de recubrimientos comestibles de gel de mucilago de penca de sábila (Aloe barbadensis Miller) y cera de carnauba, Revista VITAE, ISSN: 0121-4004, 17(3), 252-263 (2009)

Ruiz, G., Montoya, C., Paniagua, M., Degradabilidad de un polímero de almidón de yuca., Revista EIA, ISSN: 1794-1237, 12, 67-78 (2009)

Velasco, R., Enrique, M., Torres, A., Palacios, L., Rúales, J., Caracterización morfológica de películas biodegradables a partir de almidón modificado de yuca, agente antimicrobiano y plastificante, Revista de biotecnología en el sector agropecuario y agroindustrial, 10(2), 152-159 (2012) 
